\title{
Participatory Action Research as a Model for Conducting Family Research
}

\author{
Ann P. Turnbull \\ University of Kansas \\ Barbara J. Friesen \\ Portland State University \\ Carmen Ramirez \\ Austin, TX
}

\begin{abstract}
We discuss a participatory action research (PAR) approach to conducting family research. We conceptualize participatory action research as a collaborative process among researchers and stakeholders throughout the entire research sequence. Based on our five years of implementing PAR, we describe potential PAR advantages and challenges that need to be documented in future research. We propose a model of PAR implementation levels including the options of family members as research leaders and researchers as ongoing advisors, researchers and family members as co-researchers, and researchers as leaders, and family members as ongoing advisors. Finally, we discuss key implementation issues (i.e., defining stakeholders to include in the PAR process, maximizing benefits and minimizing drawbacks of diverse expertise, and addressing logistical considerations) with suggestions for effectively addressing them.
\end{abstract}

DESCRIPTORS: participatory action research, participatory research, action research, family, disability

Participatory action research (PAR) is one means of addressing the gap between researchers and the intended beneficiaries of research (McTaggart, 1991; Whyte, 1991). Participatory action research refers to a process whereby the researchers and stakeholders (those who potentially benefit from research results) collaborate in the design and conduct of all phases (e.g., specification of questions, design, data collection, data analysis, dissemination, utilization) of the research process. PAR's ultimate goal is taking action to solve the problem that is at the basis of the research (Graves, 1991; McTaggart, 1991; Whitney-Thomas, 1997). PAR is a collaborative approach to conducting research-not a discrete research methodology.

Address all correspondence and requests for reprints to Ann P. Turnbull, Beach Center on Families and Disability, University of Kansas, 3111 Haworth Hall, Lawrence, KS 66045
The National Institute on Disability and Rehabilitation Research (NIDRR) has taken a leadership role within the disability field by encouraging grantees to incorporate a PAR approach (Fenton, Batavia, \& Roody, 1993; Graves, 1991). Our conceptualization of PAR has been strongly influenced by NIDRR's definition.

Throughout the literature, many terms are used interchangeably with sometimes similar and other times conflicting definitions. These terms include participatory research, action research, participatory action research, constituency-oriented re-search and dissemination, emancipatory research, empowerment research, and discovery research. These terms all have a common focus on greater participation and influence of stakeholders in research than has traditionally characterized the research process. The approaches may differ, however, with respect to considerations such as how localized the research is to a specific context.

In this paper, we address a PAR approach consistent with NIDRR's conceptualization of researchers and stakeholders collaborating through-out the entire research process. Our focus is on families as stakeholders of research. We focus on research that is aimed at studying family life and issues important for family support. We refer to this research as family research. We (a) discuss the advantages and challenges of PAR in family research, (b) propose a range of PAR levels, and (c) raise three issues warranting creative problem solving in enhancing PAR implementation related to family research.

\section{Advantages and Challenges of PAR in Family Research}

\footnotetext{
Advantages

Although there is currently no empirical documentation of PAR advantages, we have experienced five advantages in our own implementation of PAR: (a) increased relevance of research to the concerns of family members; (b) increased rigor of research; (c) increased benefit to researchers in
} 
minimizing logistical problems; (d) increased utilization of research by families; and (e) enhanced empowerment of researchers, families, and other stakeholders. We anticipate future research that will explore these advantages empirically.

Increased relevance of research. It is perhaps selfevident that when family members are involved in identifying potential research priorities and specific topics, the research is more likely to be relevant in solving the problems that they face. Despite this obvious conclusion, the identification of research topics generally has been within the purview of researchers, and an important criterion for choosing research topics is the degree to which there exists a foundation of previous research. This way of choosing research topics results in an incremental approach to building knowledge, which may be at odds with family members' desire for immediate, useful information. (Choices of research directions based on the priority needs and concerns of families, while increasing relevance, may also fall at the margins of areas informed by previous research, requiring considerable effort to assure that necessary descriptive studies, theory-building, and measurement development are in place to support the research directions.)

Increased rigor of research. Improvements in the research process that may be associated with family involvement include increased feasibility and acceptability of research procedures, better questionnaire or interview protocol design, more accurate data, and greater longitudinal involvement with the study. In terms of feasibility of research procedures, researchers can be guided by family advice from the outset about how much time and effort families can generally be expected to invest in the research process. As collaborative decisions are made about what the researchers would like to accomplish during data collection and what the families view as feasible, our experience has been that "win-win" decisions can be made from the outset that prevent families' disgruntlement with and dropping out from the research process.

Rigor can also be increased by having PAR family collaborators not only problem-solve around how to increase recruitment and the eventual size of the participant pool, but to take on major roles in locating and informing families about the possibility of their participating in the research. Thus, sample sizes can be enhanced when families are encouraging others to participate.

Obviously, better questionnaire and interview protocol design can occur when stakeholders who are expected to interpret the items and respond have an opportunity to review and recommend appropriate protocols during the research planning stage of identifying instrumentation. Families have expressed that often their reason for not returning questionnaires has been that they find the questionnaires too confusing, lengthy, or even depressing to complete. When these viewpoints are addressed by families reviewing protocols at the outset of the planning process, the likelihood increases that families will complete the data collection protocols in a valid and reliable manner.

Finally, some families report dropping out of longitudinal studies because they are offended by procedures that do not account for their preferences, needs, and concerns. It is likely that there will be more "staying power" with longitudinal research when families find the procedures to be family-sensitive.

Increased benefit to researchers in minimizing logistical problems. Not only does increased rigor of research result from family collaboration in terms of increased feasibility and acceptability of research procedures, better questionnaire or interview protocol, more accurate data, and greater longitudinal involvement with the studies, but the problems and headaches of researchers can be decreased simultaneously. The article by Santelli, Singer, DiVenere, Ginsberg, and Powers (1998) included in this issue offers a compelling case study of how researchers benefited in recruitment from the extensive family networks of the family collaborators. Rather than spending the anticipated many months in recruitment (and then being disappointed with the sample size), researchers found that the PAR process substantially decreased the time and effort that recruitment required when families eagerly tapped their extensive networks. This same benefit can accrue for having improved instruments that result in higher completion rates.

Increased research utilization. Increased and more timely family utilization of research results is more likely to result when family members have been involved in all phases of the research process (especially identifying the research focus). It stands to reason that when families have access to information on topics that are of especially high priority to them, then they will be more likely to utilize the findings. For example, when parents whose sons and daughters with a disability are especially isolated and have no friends find useful information on how to increase the likelihood that their son or daughter will have friends, they will be more likely to use these research results than if they encounter research that has no connection to the specific problems and concerns that they are experiencing. Furthermore, when friendship is a high priority, it is likely that friendship research that has had strong family input in its planning and implementation will have greater utilization than research that has not specifically taken into account parental perspectives. This is an extremely important area for future research because to date, there has not been empirical documentation that PAR input directly contributes to increased research utilization.

Although some researchers perceive that dissemination and research utilization are an entirely different process from the research enterprise, we conceptualize dissemination and utilization as the final steps in the research process. The PAR involvement of families can help to formulate meaningful research products and can suggest ways of distributing those products so that they 
will be most accessible to families and others who support families.

Enhanced empowerment. The literature contains many assertions that PAR contributes to empowering participants on both a conceptual and applied level (Barnes, 1992; Lather, 1986; McTaggart, 1991; Sample, 1996; Yeich \& Levine, 1992; Zarb, 1992). We have found a number of empowering outcomes of the PAR process, including family members taking action to get what they want and need, families having increased opportunities for contribution and input, researchers having a significant learning opportunity about the reality of family life and the nature of family support, and researchers and families expanding their sense of collective power through their collaboration with each other.

Carmen Ramirez, a grassroots family advocate (and TASH Board member), describes the empowerment that she has experienced from her participation on a PAR Committee:

In dealing with competent and caring researchers, I have confirmed as a parent and family advocate that I have much to offer. As I am increasingly involved in PAR research, I realize that my experience as a parent of a child with a disability is invaluable, because it is this experience that translates into information which others, parents and professionals alike, can utilize to foster the inherent strengths of families. Because the needs of Latino families have long been ignored, as a participant in this research my hopes are that some of our needs and attributes will be addressed as a result of earnest and genuine collaboration between parents and researchers.

\section{Challenges}

Five challenges that we have encountered in terms of using PAR are (a) increased time in planning and conducting research, (b) researchers feeling criticized by parents, (c) increased funding required to cover PAR costs, (d) the lack of family homogeneity, and (e) increased need to change institutional rules.

Increased time. Every kind of collaborative problem-solving approach requires time. In the case of PAR, time is needed for many additional tasks including locating families and other stakeholders who can advise on a project, arranging the logistics of communication, carrying out the communication, allowing sufficient time in advance of the communication for preparation by families, and allowing sufficient time after the communication for adequate reflection and feedback. Because grant announcements come out on a short response schedule, it is exceedingly difficult to incorporate the additional time that PAR requires.

A particularly critical element related to time (for which we were not prepared) is the amount of time needed to develop trusting relationships with families who have had negative experiences with researchers. We have found this especially to be true in developing research collaboration with families from culturally and linguistically diverse backgrounds who may view research as exploitative (Markey, Santelli, \& Turnbull, in press). A Latino parent expressed her perception of the research process as follows: "Researchers come in, take information from us, and use it to their own advantage. What's in it for us?" When this is the perspective of families who are invited into a PAR process, it can take 1-2 years of ongoing communication before trust is at a sufficient level for a genuine partnership to evolve. Although this heavy time investment on the front end of the research partnership can lead to advantages on a long-term basis, it is, nevertheless, a tremendous time investment.

Researchers feeling criticized by parents. Given that some families have negative views of research, researchers implementing PAR may be surprised by family members "letting off steam" about some of their perspectives about research being exploitative. It can be challenging for re-searchers who have good intentions about PAR to be the "targets" for negative debriefing. Becoming defensive can be an understandable reaction.

Additionally, despite many efforts related to being sensitive and responsive, the communication gap between researchers and family members who do not have research training can be substantial. Sometimes the communication gap is created by different values; in other situations, family members may be offended by questions or ideas when they infer meaning that was not intended. A frequent example is when family members interpret questions meant to elicit descriptive data as normative, implying that they and their family should do whatever the question asks about. This sensitivity is entirely understandable because of family members' history of feeling blamed or criticized in professional settings (Turnbull \& Turnbull, 1985), but this may not emanate directly from their experiences with researchers.

Additionally, family members may have negative attitudes about research and researchers, whether they have had bad experiences or not. Many families recognize the extreme shortage of funding for services and wonder about the rationale for spending money on research. As we will address later, mutual education of researchers and families is critically important.

Increased funding. PAR can increase the costs of research because additional people are involved who warrant payment. In some cases, it has been traditional to invite families to advise on projects but to not reimburse them for their time or to provide a very small reimbursement. Increasingly, state-of-the-art PAR processes encourage the reimbursement of families for their time and expertise throughout the entire collaborative process. When families are paid at comparable rates to other consultants and/or researchers, it becomes readily apparent that all of the time and effort invested in PAR meetings, reviewing 
protocols, recruiting families, and copresenting at conferences can he expensive.

Lack of family homogeneity. Family priorities often vary according to the age of the family member with a disability, the particular nature of the disability, and family system characteristics. For example, frequently families who have a son or daughter with challenging behavior express an urgent need for assistance in knowing how to minimize the challenging behavior and replace it with more appropriate behavior. This topic, however, is not a priority of families whose son or daughter does not experience challenging behavior. Thus, it cannot be assumed that there are certain topics that are relevant to all families and other topics that are irrelevant. Thus, a major PAR challenge is addressing discrepancies among families of what they consider to be important research to pursue.

Increased need to change institutional rules. There are a number of major barriers to using a PAR process within the university structure. For example, some PAR advisors note the incomprehensibility of consent forms and urge researchers to draft new forms that meet the legal requirements but that are written so that people can readily comprehend them. Human Subjects Review Committees often find this troubling, but we have found that there are ways to meet simultaneously the needs of the Review Committee and the need for comprehensibility by carefully redrafting forms with the collaborative input of people with expertise in the legal requirements that the forms must meet and family input addressing clarity and comprehension. (Principles of adult learning such as spacing, headings, advanced organizers, and tone all make a difference in families' ability to comprehend the information).

Other institutional barriers involve providing advance payments to PAR advisors so that they can travel to conferences and copresent papers. As we address later in the paper, many PAR advisors do not have credit cards or the available cash to wait for a reimbursement, which the university rules prefer.

\section{Levels of Family Participation in Research}

Implementing PAR requires attention to the relative distribution of decision making between researchers and members of relevant stakeholder groups. In Figure 1, we present six levels of family participation that illustrate an ever-increasing extent of family decision making related to the research enterprise, adapted from the work of Arnstein (1969) and Bailey (1994).

Level 1, reflecting minimal involvement and no decision making, is the role of families as research participants. Levels 2 and 3, family members as advisory board members and family members as occasional reviewers and consultants, entail increasing levels of involvement, but relatively little influence with regard to the design and implementation of the research plan. Because family members have little or no influence regarding the purpose, content, or direction of the research at these first three levels, they fall short of the definition of PAR (collaborative decision making among researchers and stakeholders). Traditional practices within the research community have largely involved families and other stakeholders at one of these three levels.

The goal of PAR is to move in the direction of family decision making. Levels 4-6 represent various degrees of PAR implementation. These include researchers as leaders and family members as ongoing advisors (level 4), family members as coresearchers (level 5), and family members as research leaders with researchers serving as ongoing advisors (level 6). Many ways exist for implementing these three levels, and each style of implementation raises specific issues. Most of our experience has been at level 4 researchers as leaders and family members as ongoing advisors, although we have also been involved in several research projects where family members, have been coresearchers (level 5).

PAR is an approach that can be used with any research methodology. Although many people may equate PAR with qualitative research, it is equally applicable to other research methodologies. For example, one of the new areas of emphasis in IDEA '97 is the use of mediation as a strategy for resolving conflict between parents and educators. The Office of Special Education Programs (OSEP) intends to fund a large technical assistance center on dispute resolution (U. S. Department of Education, 1997). This new center will likely launch a long-term research initiative in mediation. In the process of submitting this proposal, researchers might identify parents with a variety of experiences and interests in mediation-those (a) who have had full due process hearings as well as nonadversarial forms of dispute resolution, (b) who provide training on educational advocacy through Parent Training and Information Centers, (c) who represent a broad range of cultural and linguistic diversity, and (d) who have children of varying ages and varying types of disabilities.

This group of parent advisors might come together with researchers to form a PAR committee with the goal of specifying research questions and planning the details of research implementation and utilization. Some of the studies planned might be of a quantitative nature, whereas others might involve single-subject analysis and/or policy analysis. Whatever the methodology, the PAR committee for conceptualizing and writing the proposal for this new center might choose the level at which they are going to collaborate in making decisions. In fact, they might use different levels of decision making for different studies. 


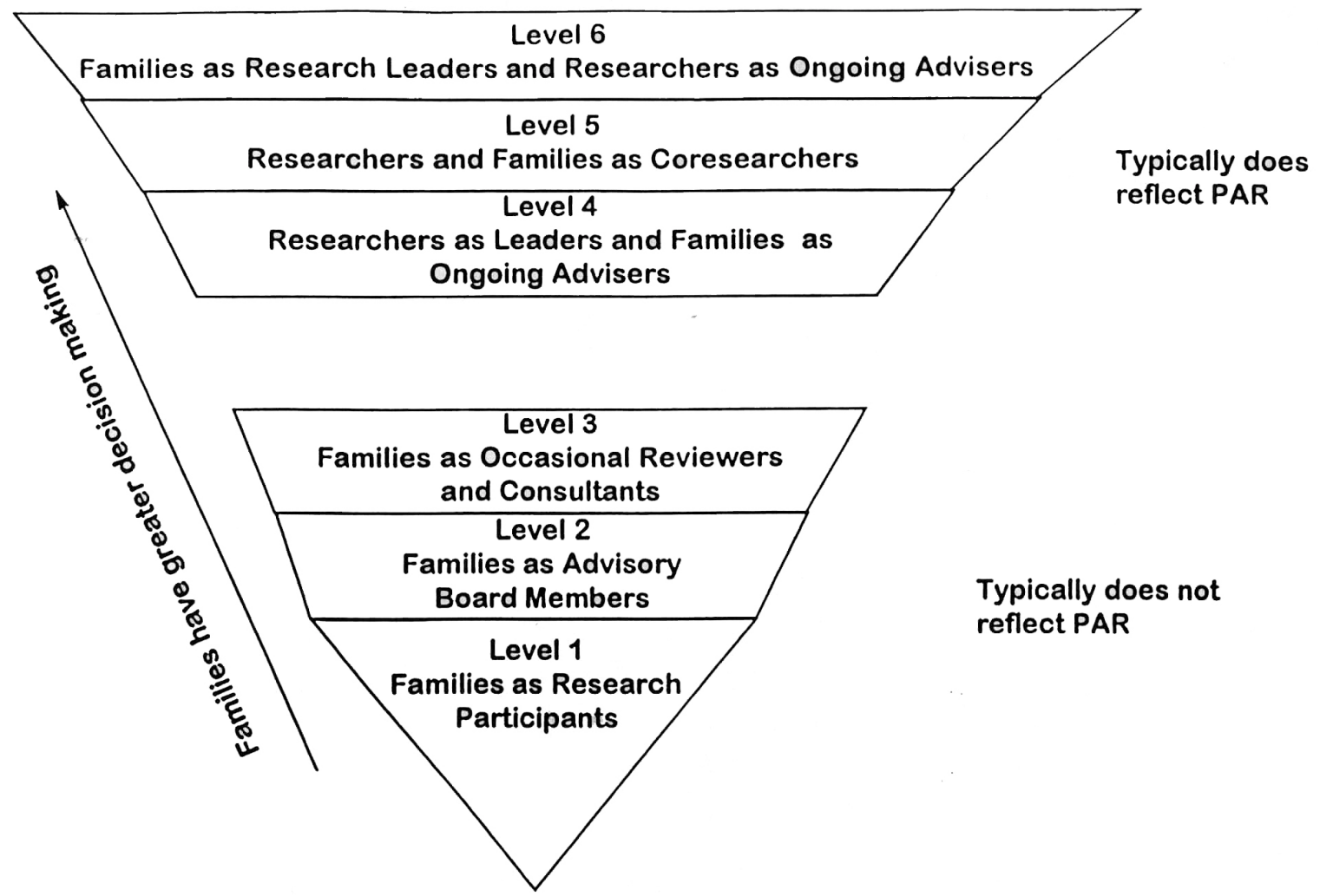

Figure 1. Continuum of family participation in research.

One of the studies initiated could be a national survey of parents and educators who have participated in mediation during the last three years to identify the benefits and drawbacks from their perspectives. The particular PAR committee for this study might include parents and educators, who have been through mediation, working with researchers. Working as coresearchers (level 5), the parents and educators could be helpful to the research team in generating a description of the benefits and drawbacks from their own perspectives. The researchers could be extremely helpful to the parents and educators in assisting them in codifying their experiences by determining major themes and sub-themes. This codification could lead toward the development of instrumentation. Working together as a PAR committee, the researchers, families, and educators could reflect on the appropriate timing of data collection with the parents and educators helping the researchers to understand how some of their perspectives may have stayed the same or changed across different time intervals after the mediation was concluded.

As an alternative, another study recommended for this proposal might include a policy analysis of state statutes and regulations pertaining to mediation. This study might have a PAR committee comprised of parents who have leading advocacy roles at the state level, the governmental affairs chairpersons of state family organizations, and state special education administrators. The families and administrators could assist the researchers in developing a protocol for the policy review. The family and administrator stakeholders might also cosign a cover letter to their counterparts in states encouraging them to cooperate with the research team in providing information and in sharing their state documents.

A major consideration is that the specific nuances of each study should guide who the PAR advisors are and should shape the appropriate level at which the PAR process will be implemented. There is no cookbook approach for PAR implementation. An important consideration will be the researchers' comfort and experience. It may be that many researchers who are accustomed to working at levels 1-3 will be interested in trying level 4 . Once they become more comfortable with families and other stakeholders as ongoing advisors, in the future they may be interested in thinking about extending that collaboration to families being coresearchers. Researchers do not need to feel as if they are in a forced-choice situation of starting at level 5 or 6 or rejecting PAR. In fact, most families are not interested in being the research leaders (level 6), 
although some families, as they participate increasingly in PAR, will want to try this level as well.

Another consideration in choosing the appropriate level for implementation will be the funding agency. Some funding agencies encourage the use of PAR, whereas for other agencies PAR might be considered an inappropriate process. The unique features of each situation need to be considered.

Thus, PAR has different manifestations, with the bottom line of each being strong collaboration among researchers and families so that the expertise that each brings carries equal or at least meaningful weight. PAR's collaborative decision making is also proactive, whereas practices at levels 1-3 often reflect reactive commenting or review of decisions that researchers have made previously.

\section{Issues in PAR Implementation Within Family Research}

In this section, we address three issues warranting creative problem solving in enhancing the quality and quantity of PAR implementation related to family research. These issues include (a) defining stakeholders, (b) achieving a balance of expertise, and (c) addressing logistical considerations.

\section{Defining Stakeholders}

PAR definitions refer generally to the need for stakeholders to participate in all research phases. Determining who the stakeholders are in family research raises a number of issues including (a) the definition of family and (b) selecting people, other than family members, who may be beneficiaries of family research.

Definition of family. Family is defined in diverse ways both within and outside of the disability field (Doherty, Boss, LaRossa, Schumm, \& Steinmetz, 1993; Popenoe, 1988). We define family as two or more people who carry out the responsibilities that families typically perform and who regard themselves as a family regardless of having a blood or marriage relationship. This definition is open-ended and honors cultural and individual diversity.

We believe that more affirmative efforts need to be made to ensure that families are represented from a range of gender, racial/ethnic, and socioeconomic backgrounds. In a review of four leading early childhood journals (i.e., Journal of Early Intervention, Topics in Early Childhood Special Education, Infant/Toddler Intervention Journal, and Infants and Young Children) in 1992-1993 over a 12-month period, articles were identified that had "parent" or "family" in the title (Turnbull \& Turbiville, 1993). In the 11 articles that had one of these words in the title, the sample population in the research included a 12:1 ratio of mothers to fathers. Approximately $75-80 \%$ of the research participants were Euro-American. The field should not make the same mistake (i.e., assuming that "parent" or "family" means Euro-American mothers) in defining families for PAR participation that it has made in defining families for participation in family research. Especially when conducting research with culturally diverse families, the culturally appropriate definition of family needs to be honored.

In many native American Indian families, the childrearing activities may rest with other family members.... In fact, in some tribes, the uncles instead of the parents may provide most of the discipline, while grandparents provide most of the spiritual guidance and teaching. (Joe \& Malach, 1992, p. 106)

An additional consideration is that any family member is often considered generic enough to represent all family issues. For example, in a research study focusing on families who have engaged in abuse and/or neglect of their children, is it sufficient for a family member who uses empowering and respectful discipline to be considered a stakeholder? We recommend that the tremendous diversity of families be respected and that PAR membership particularly seek families whose experiences and perspectives can inform the specific nuances of each research study. Thus, rather than any family member being inter-changeable with any other one, PAR selection should be customized to match the rich experiential and cultural perspectives of families who are anticipated to benefit from the research results in terms of solving the priority problems and concerns that they face.

People other than family members. Families are not the only participants or stakeholders of family research. For example, family research often shows reports of data from service providers about their interactions with families. Furthermore, some family research may not involve a particular stakeholder group as research participants, but that group's perspectives may be very important in enhancing the feasibility of research utilization. For example, a research study focusing on parent collaboration in school reform efforts may only gather data from parents. If teachers and administrators were also included on the PAR committee, they could likely identify issues they think should be addressed for enhanced parent collaboration to be implemented systemically. Involving teachers and administrators recognizes that parent collaboration occurs within a context that must also be considered.

Increasing the range of stakeholders in the research process may increase its social validity (Schwartz \& Baer, 1991). It also, however, increases the complexity of research planning and is likely to raise the extent to which conflicts must be managed. There are often discrepancies in the ways that families and professionals define problems and needed services (Bernheim \& Switalski, 1988; Spaniol, Jung, Zipple, \& Fitzgerald, 1987), assess the process of working together (Hermary \& Rempel, 1990), and define appropriate roles for families and professionals (Chavkin \& Williams, 1985). 
For example, studying families whose children had serious physical disabilities, Blackard and Barsh (1982) found that professionals overestimated the negative impact of coping with the child's disability and underestimated family abilities.

A concern also arises that by involving a wider range of stakeholders, it can be fairly easy for family voices to be drowned. For example, if a PAR committee in family research involved two persons from each of the following groups-parents, individuals with disabilities, teachers, administrators-parents would have only $25 \%$ of the participation, although they are considered the primary stakeholders. Including stakeholders broadly with-out minimizing family input requires carefully weighing benefits and drawbacks.

\section{Achieving a Balance of Expertise}

Crafting successful family-researcher partner-ships requires attention to three important needs: (a) Family members and researchers must learn to identify and respect the expertise that the other brings; (b) there must be mechanisms for the mutual education of all parties in the research endeavor; and (c) the process for making decisions must be clearly identified.

Identifying and valuing expertise. Learning to appreciate the expertise that the other (e.g., researcher, family member) brings is fundamental to establishing a respectful partnership; it also takes attention and effort. For researchers, a central issue is to understand the value of the rich histories, experiences, and perspectives that family members bring by virtue of their personal, family, and cultural situations. This appreciation, however, may not come easily to some researchers. For example, a couple of years ago one of us participated with a national research agency in planning for a longitudinal research study focusing on intervention with primarily low income, African American, single mothers. Consistent with traditional practices, the research planning team included only researchers-most of whom were Euro-American males, and all of whom had terminal graduate degrees. It is likely that the individuals who convened this group never considered inviting low-income, African American, single women to share the personal and systemic barriers that stood in the way of their providing quality care for their children. Likely, these mothers could have shared a great deal of information that would lead to the specification of relevant variables that may have gone beyond the traditional measures that are typically used in similar studies. Furthermore, by having these mothers provide guidance on the types of protocols that they would or would not be willing to complete, it is likely that the researchers could have enhanced the size of their sample and the return rate of the sample of the various research instruments. It is impossible, however, for researchers to accrue this kind of beneficial advice at the outset of planning if they do not value the expertise and insights of the research participants. Valuing others' expertise, particularly in situations characterized by cultural and linguistic diversity, requires researchers to acknowledge the limitations of their own perspectives and to be open to diverse points of view.

The issue of establishing relevant expertise is apparent in recent efforts by some federal agencies to include individuals with disabilities, family members, service providers, and other stake-holders in the peer review process for research proposals. (Collaboration in peer review can be conceptualized as a PAR process for identifying the research that should be funded.) Although involving stakeholders in peer review has been experienced by some participants as awkward and unsatisfactory, our view is that the process will be improved by clarifying the relevant expertise and expectations of all peer reviewers. Although a process of preparing stakeholders for PAR committees and/or the peer review process should include a basic orientation to research purposes and procedures, it is unreasonable to expect families and other stakeholders to make recommendations about many aspects of research design, measurement, and statistical procedures. (Likewise, researchers' expertise typically is limited to certain types of research designs. Quantitative researchers may have limitations similar to family members when commenting on qualitative designs. Thus, the concept of relevant expertise applies equally to participants from all backgrounds.)

Family members have a great deal to offer PAR committees and/or peer review teams with regard to issues such as whether the research topic and questions are relevant to family concerns, whether proposals include consideration of families' strengths as well as their needs, and whether research and dissemination plans are feasible. Our experience with research planning and peer review processes leads us to recommend that clear statements about the relevant expertise and expectations of family members and practitioners, as well as those of researchers, be clearly stated. This allows responsibility for aspects of the review to be assigned, and appropriate training and support for committee members to be provided.

Mutual education. Approaches to the mutual education of researchers and family members are limited only by the imagination of the research team. At the beginning of the research process, we believe it is important to take the time to discuss areas where family members and researchers may have different values or priorities and to establish common goals and expectations for the collaboration. As mentioned earlier, one of the most apparent differences between family members and researchers is often their orientation to time. Family members often want research results that can be applied immediately to improve the lives of their families and others. One family member who was part of a group to develop a 5-year family research agenda commented, "Your 5-year plan is my child's adolescence!" Researchers, on the other hand, often see a single study as one of a series or emphasize the importance of 
longitudinal investigations. Next we include strategies for providing education to researchers and families to enhance the likelihood of successful PAR practices.

For researchers:

1. Faculty and staff can benefit from learning firsthand about the lives of families who include persons with disabilities. Interacting and communicating with families from culturally and linguistically diverse backgrounds, we believe, should receive special priority. These can include:

- informal social contacts (lunch, or a Center pizza feed);

- verbal or written presentations by family members;

- collaboration training specifically designed to improve communication and explore value positions;

- feedback sessions designed to capture particular aspects of family concerns, e.g., "The most important issue that I face with regard to transition is...."

- books, monographs, and other first-person accounts of the experience of family members and persons with disabilities; and

- provision of respite care for families.

2. Many family members also have professional degrees and research expertise, and these individuals can perform an important bridging and translation function as part of research teams and peer review committees. They can provide a unique perspective that is helpful to both researchers and other family members; but it is also important to remember that they may not necessarily represent the perspectives of families who do not also have professional preparation.

3. Researchers may need assistance in learning how best to communicate with family audiences about the purposes, methods, and results of research. At a conference featuring family-centered programs and family research issues at the Research and Training Center on Family Support and Children's Mental Health, the participation of family members and youth in the research to be presented and in the presentation itself are some of the criteria by which proposals for workshops and paper presentations are evaluated. In preparation for the conference, research presenters are provided with a list of tips about adapting their presentations to family members, service providers, and administrators. Technically oriented presentations are marked on the conference program so that all conference participants may more easily identify sessions of interest.
For family members:

1. An orientation about the fundamental purposes and procedures of the research process can help family members prepare to become active partners in research. One strategy is to conduct training sessions for family members and other PAR participants who do not have research expertise. Handouts such as glossaries of research terms, design flow charts, and other summary materials are often useful.

2. Colleges and universities, as well as community adult education programs, might offer regular college courses, short courses, correspondence courses, or other varieties of content presentation on research issues for the benefit of family members and other stakeholders (e.g., administrators, service providers). Family leaders of family support organizations might be particularly interested in such courses, if they could be applied toward an undergraduate or graduate degree. These family leaders are likely to be ones who might be involved on an ongoing basis in PAR collaboration. It would be particularly appropriate to consider various distance-learning options to enhance the convenience of the research training to family schedules and time constraints.

3. Similar to good planning strategies for any meeting, information might be sent out to all PAR advisors in advance of meetings so that they will have an opportunity to review the documents and identify any questions or issues around which follow up would be desirable. Additionally, before meetings research staff might telephone the PAR family members to answer questions in order to increase the comfort level of the family participants and increase their level of preparation. For example, if particular research instruments, questionnaires, or protocols are going to be discussed at a meeting, it could be extremely beneficial for family participants to have these in advance so that they can comment on factors such as their clarity, readability, and focus on relevant dimensions.

Clarifying the decision process. Establishing clear expectations about how researchers and family members will work together includes being specific about how decisions will be made. It is important to address the issue of ultimate responsibility early to avoid misunderstanding later in the process. This can depend on the purpose of the research, the auspice and funding, who initiated the research, and the attitudes and philosophy of the participants. The ultimate responsibility for decision making depends upon the level at which PAR is being implemented (see Figure 1). If family members are taking the lead and driving the process (illustrated in level 6 of Figure 1), they will retain ultimate decisionmaking authority. Other options, as illustrated in Figure 1 , include researchers and family members as co- 
researchers (level 5) where a consensus decision-making process might be appropriate; and researchers as leaders and family members as advisors (level 4) where it is obvious that researchers would retain considerable control over the research process.

One decision-making approach is to return to the question of relevant expertise, identifying some issues as more within the realm of researcher expertise, having researchers make recommendations for family members to endorse, or not, depending on their background and preferences. Similarly, other issues may be seen as lying primarily within the expertise of families, and they may make initial recommendations to the re-searchers. The underlying principle here is to acknowledge the diverse strengths and contributions of all involved. This principle is the essence of collaboration. Thus, within a single PAR committee working on a single research study, there might be opportunities to move back and forth between levels 4-6 depending upon the particular decisions that need to be made.

\section{Addressing Logistical Considerations}

As we have discussed previously, effective implementation of PAR practices is highly demanding of time and fiscal resources (Hall, 1984). Building a sense of cohesion not only with the research staff but with other members of the PAR committee can require a number of contacts over an extended period of time. Similarly, creating trust and greater openness for candid and direct input can require extensive contacts and large time periods.

Creating cohesion and trust is typically fostered best through face-to-face meetings. Increasing the diversity of membership representing different regional and cultural perspectives, however, may actually reduce the opportunity for face-to-face PAR meetings (e.g., PAR committee members bring a diverse geographical perspective, but they are separated by great distances and driving to a meeting is not feasible). This means that most communication occurs through telephone conference calls, individual telephone calls, e-mail, and correspondence.

Funding agencies have a significant leadership role in addressing PAR logistical barriers. For example, writing a proposal with significant PAR input requires extensively more time than preparing proposals in a singular and isolated manner. Given the amount of notice that exists between a competition announcement and the proposal due date, it is practically impossible to meet the letter and spirit of the PAR process. Furthermore, it may be necessary to reduce the scope of projects to have time for meaningful PAR input. Time and effort invested in quality PAR practices will need to come from somewhere other than sheer additional work hours on the part of researchers, families, and other stakeholders. Although some people might raise an objection to reducing the scope of research to have time for PAR, if PAR practices result in substantially greater dissemination and utilization of research, a greater ultimate impact may be made in solving problems that families and other stakeholders face. One of the tremendous losses in the past has been the fiscal and personnel investment in research that has not at all been adequately disseminated and utilized to solve the real and frequent problems faced by families and other stakeholders (Bruyere, 1993; Carbine, 1997; Fuchs \& Fuchs, 1990; Hoshmand \& Polkinghorne, 1992; Kaufman, Schiller, Birman, \& Coutinho, 1993; Lovitt \& Higgins, 1996; Turnbull \& Ruef, 1996).

PAR involvement also has time and fiscal implications for families and for other research stakeholders. Family members must either take time off from work or commit time during evening hours. The costs involved with mailing letters and packages, making telephone calls, and generally participating with the researchers, need to be covered by the research institution. The issue of payment for time, however, is a critically important one to address. One option is to incorporate PAR stipends or vouchers for resources into research budgets. (Some families may be uncomfortable accepting monetary payment. One situation we encountered concerned families from a community characterized by a high degree of poverty. The PAR committee advised us that families would have no place to get a check cashed. In this situation, we were able to buy vouchers to an indigenous community grocery story that families could readily use in lieu of a check. These types of alterations are not typically handled within university reimbursement procedures.) Another fiscal consideration is that families may need advance payment to come to a meeting rather than to wait for reimbursement. Thus, it is necessary to work with university offices in arranging for advance payments of major expenses (e.g., airline ticket, hotel), in addition to providing advance funds for smaller expenses such as taxi fares. One cannot only look at the costs, however, without also looking at the savings related to PAR. From our experience, family collaboration has extensively reduced the time and effort in locating participants for research studies, organizing conferences, and gaining credibility with research participants, particularly ones from diverse cultural and linguistic backgrounds. Thus, there can be a tremendous savings of time and fiscal resources that need to be documented carefully through cost-benefit studies.

\section{Summary}

This article reflects some of our perspectives on PAR based on our experience of implementing it over the past five years. In conceptualizing PAR as a collaborative process between researchers and the people who are expected to benefit from the research, we have experienced a number of advantages and challenges. To date, our focus has been primarily in trying to "figure out" how to implement PAR in an authentic and comprehensive manner. We recognize a major need for systemic evaluation to document the potential advantages 
and challenges that we have raised in this article. We hope that this evaluation will include extensive case studies as well, to identify "what works" in ongoing implementation within singular research studies, as well as throughout all aspects of research centers. We believe that the field is in its early infancy in understanding the potential of PAR collaboration, and we look forward with enthusiasm to the next phases of inquiry in establishing efficacy and best practices.

\section{References}

Arnstein, S. R. (1969). A ladder of citizen participation. Journal of the American Institute of Planners, 35, 216-224.

Bailey, D. (1994). Organizational empowerment: From self to interbeing. In L. Gutierrez \& P. Nurius (Eds.), Education and research for empowerment practice (pp. 37-44). Seattle: University of Washington, School of Social Work, Center for Policy and Practice Research.

Barnes, C. (1992). Qualitative research: Valuable or irrelevant? Disability, Handicap, \& Society, 7, 115124.

Bernheim, K. F., \& Switalski, T. (1988). Mental health staff and patients' relatives: How they view each other. Hospital and Community Psychiatry, 39(6), 6368.

Blackard, M. K., \& Barsh, E. T. (1982). Parents' and professionals' perceptions of the handicapped child's impact on the family. Journal of the Association for the Severely Handicapped, 7(2), 62-70.

Bruyere, S. M. (1993). Participatory action research: Overview and implications for family members of persons with disabilities. Journal of Vocational Rehabilitation, 3(2), 62-68.

Carnine, D. (1997). Bridging the research-to-practice gap. Exceptional Children, 63(4), 513-521.

Chavkin, N. F., \& Williams, D. L. (1985). Parent involvement in education. School Social Work Journal, 10, 35-46.

Doherty, W. J., Boss, P. G., LaRossa, R., Schumm, W. R., \& Steinmetz, S. K. (1993). Family theories and methods: A contextual approach. In Sourcebook of family theories and methods: A contextual approach (pp. 3-30). New York: Plenum Press.

Fenton, J., Batavia, A., \& Roody, D. (1993). Proposed policy statement for the National Institute on Disability and Rehabilitation Research on constituency-oriented research and dissemination (CORD). Washington, DC: U. S. Department of Education, National Institute on Disability and Rehabilitation Research.

Fuchs, D., \& Fuchs, L. S. (1990). Making educational research more important. Exceptional Children, 57, 102-107.
Graves, W. H. (1991, September). Participatory action research: A new paradigm for disability and rehabilitation research. ARCA Newsletter, 19, 9-10.

Hall, B. (1984). Research, commitment, and action: The role of participatory research. International Review of Education, 30, 289-299.

Hermary, M. E., \& Remple, J. (1990). Parental and staff perceptions of individual programming teams: Collaboration in and beyond the conference. Education and Training in Mental Retardation, 25, 25-32.

Hoshmand, L. T., \& Polkinghorne, D. E. (1992). Redefining the science-practice relationship and professional training. American Psychologist, 47(1), 55-66.

Joe, J. R., \& Malach, R. S. (1992). Families with Native American roots. In E. W. Lynch \& M. J. Hanson (Eds.), Developing cross-cultural competence: A guide for working with young children and their families (pp. 89-119). Baltimore: Paul H. Brookes.

Kaufman, M., Schiller, E., Birman, B., \& Coutinho, M. (1993). A federal perspective on improving practices, programs, and policies in special education. Evaluation and Program Planning, 16, 263-269.

Lather, P. (1986). Research as praxis. Harvard Educational Review, 56, 257-277.

Lovitt, T. C., \& Higgins, A. K. (1996). The gap: Research into practice. Teaching Exceptional Children, 28(2), 64-68.

Markey, U., Santelli, B., \& Turnbull, A. P. (in press). Participatory action research involving families from underserved communities and researchers: Respecting cultural and linguistic diversity. Multicultural Voices.

McTaggart, R. (1991). Principles for participatory action research. Adult Education Quarterly, 41, 168-187.

Popenoe, D. (1988). Disturbing the nest: Family change and decline in modern societies. New York: Aldine de Gruyter.

Sample, P. L. (1996). Beginnings: Participatory action research and adults with developmental disabilities. Disability \& Society, 11, 317-332.

Santelli, B., Singer, G. H. S., DiVenere, N., Ginsberg, C., \& Powers, L. E. (1998). Participatory action research: Reflections on critical incidents in a PAR project. The Journal of The Association for Persons with Severe Handicaps, 23(3), 211-222.

Schwartz, I. S., \& Baer, D. M. (1991). Social validity assessments: Is current practice state of the art? Journal of Applied Behavior Analysis, 24, 189-204.

Spaniol, L., Jung, H., Zipple, A., \& Fitzgerald, S. (1987). Families as a resource in the rehabilitation of the severely psychiatrically disabled. In A. B. Hatfield \& H. P. Lefley (Eds.), Families of the mentally ill: Coping and adaptation (pp. 167-190). New York: Guilford Press.

Turnbull, A. P., \& Ruef, M. B. (1996). Family perspectives on problem behavior. Mental Retardation, 34, 280-293. 
Turnbull, A. P., \& Turbiville, V. P. (1993, September). Fathers' roles in intervention pro-grams for children at special risks: Disabled, chronically ill, and children living in poverty. Paper presented at the National Research Council, National Academy of Science, Washington, DC.

Turnbull, A. P., \& Turnbull, H. R. (1985). Parents speak out: Then and now (2nd ed.). Upper Saddle River, NJ: Merrill/ Prentice Hall.

U. S. Department of Education, Direct Grant Programs and Fellowship Programs, 62 Fed. Reg. 52430 (1997).

Whyte, W. F. (Ed.). (1991). Participatory action research. Newbury Park, CA: Sage.

Whitney-Thomas, J. (1997). Participatory action research as an approach to enhancing quality of life for individuals with disabilities. In R. L. Schalock (Ed.), Quality of life: Vol. H. Application to persons with disabilities (pp. 181-197). Washington, DC: American Association on Mental Retardation.

Yeich, S., \& Levine, R. (1992). Participatory research's contribution to a conceptualization of empowerment. Journal of Applied Social Psychology, 22(24), 18941908.

Zarb, G. (1992). On the road to Damascus: First steps towards changing the relations of disability research production. Disability, Handicap, \& Society, 7(2), $125-138$

Received: May 15, 1997

Final Acceptance: March 15, 1998

Guest Editor in Charge: Luanna

Meyer

This research was supported by a grant from the National Institute on Disability and Rehabilitation Research to the Beach Center on Disability, Grant \#H133B980050. 